\title{
Continuity and enhancement of quality management during commissioning of W7-X
}

\author{
Reinhard Vilbrandt, Hans-Stephan Bosch, Jost-Henrich Feist, Thomas Klinger
}

Max-Planck-Institute for Plasma Physics, D-17491 Greifswald, Germany

\begin{abstract}
The commissioning of Wendelstein 7-X, the first numerically optimized stellarator, is a new phase in the project. The general planning and execution of the commissioning of the entire W7-X system follow its structure into appropriate components, subsystems and systems. The approach for taking these systems into operation will lead to so called local commissioning which is usually executed for individual systems in connection with the necessary peripheral devices and auxiliary systems. The subsequent step-wise testing and commissioning of the systems in connection with the central device of W7-X, including the central safety control, and the central data acquisition system is performed in the second step, the so called integrated commissioning. This leads directly to the preparation of first plasma operation. New organizational and quality management elements have been added to the running system, or existing, proven tools have been modified. The major new organizational structures and tasks and the quality planning and assurance tools are described in more detail. The experience during the first commissioning steps of the vacuum and cryogenic systems is outlined.
\end{abstract}

Keywords: Commissioning, Quality, Risk Assessment, Magnet System, Stellarator

\section{Introduction}

With the completion of the Wendelstein 7-X (W7-X) assembly and its peripheral systems, all components, subsystems and systems (summarized as system in the following) will be subject to final testing and commissioning. For the preparation of this new phase of the project, in January 2013 an international workshop was held to learn the experience gained in other organizations and projects [1-3] like CEA, CERN, ITER, JET, NIFS, PPPL, NFSI/KSTAR [4]. Based on the good experience with the quality management of the project, which is certified according to DIN ISO 9001, the new elements became inherent part of this system. As a result a process instruction was set up, which regulates the organizational subjects, the technical approach, the preparation of necessary documents, and the execution of commissioning.

Due to its complexness, the many interdependencies and limiting conditions, the commissioning has to be planned and executed with great care and accuracy. Responsibilities and competences have to be defined clearly. Personnel must not be exposed to any hazards, and technological risks have to be minimized. The commissioning shall be done efficiently and their final results shall reflect the currently acknowledged state of science and technology.

For the most efficient management of related processes and interactions between the persons involved, procedures had been set up, which will optimize the execution of the work, the systematic checking of the implementation of set targets, the preparation of comprehensible documentations, and the validation of the results achieved.

\section{General approach}

The general planning and execution of the commissioning follow the system structure of W7-X. For the coordination of the necessary work between these systems, the work breakdown structure WBS (Section 4.1) is used.

This approach leads to so called local commissioning for individual systems. The subsequent combined testing and commissioning of the separate systems in connection with the central device of W7-X is called integrated commissioning.

Each commissioning must be executed only after completed assembly of the respective systems. This is proven by the complete execution of the corresponding Quality Assurance and Assembly Plan (QAAP) [5]. The correct state of assembly together with its proper documentation is to be checked prior to each commissioning. Remaining work has to be transferred into the commissioning assurance template (CAT) (Section 4.4).

The requirements for the execution of function tests (hardware, software and other systems) must be analyzed in detail. Fulfillment of aforementioned conditions must be proved prior to start of the actual commissioning.

Operation instructions must be prepared for each system. They contain the description of all relevant technical details, a description of the intended use of the system and instructions for the putting into service (Section 4.2).

During execution of commissioning, safety systems such as emergency shut-down, stress release and emptying systems or safety control units have to be checked actively. That also includes training and tests of safeguard and control measures. 
Personnel and device safety are very important especially during commissioning. Because the system is new, and related experience does not really exist in this situation, it is indispensible to take special care for this. Commissioning may involve additional particular hazards compared to the risks in the later operation phase. To exclude or minimize hazards, appropriate and sometimes additional temporary safety precautions must be implemented (Section 4.3).

For each commissioning the responsible officer or project leader (RO) has to prepare a commissioning assurance template (CAT) (section 4.4) as commissioning instruction, which contains all necessary work and test steps in the right sequence. Test protocols and check lists serve to verify exact examinations.

Based on the instruction manual, the CAT and the safety analysis the responsible for the personnel safety and the torus hall shift leader(s) prepare a special health and safety briefing (Section 4.3).

Each commissioning is be completed by a report, which summarizes the outcome, the unexpected deviations, the observations, experiences, and proposals for improvement and modifications.

Although putting into service is a step which is formally to be separated from commissioning activities, it will be prepared and executed like an integral commissioning and always with due regard to its complexity.

\section{Organization and management}

The W7-X Board under the leadership of the project director is the central and responsible panel for the commissioning. (Fig. 1). The W7-X Board appointed the responsible Chief Commissioning Manager (CCM), who is in personal union the division head of $\mathrm{W} 7-\mathrm{X}$ operations. He coordinates all activities for the commissioning. The division head assembly supports him coordinating simultaneously ongoing (last) assembly activities in the torus hall and other assembly areas.

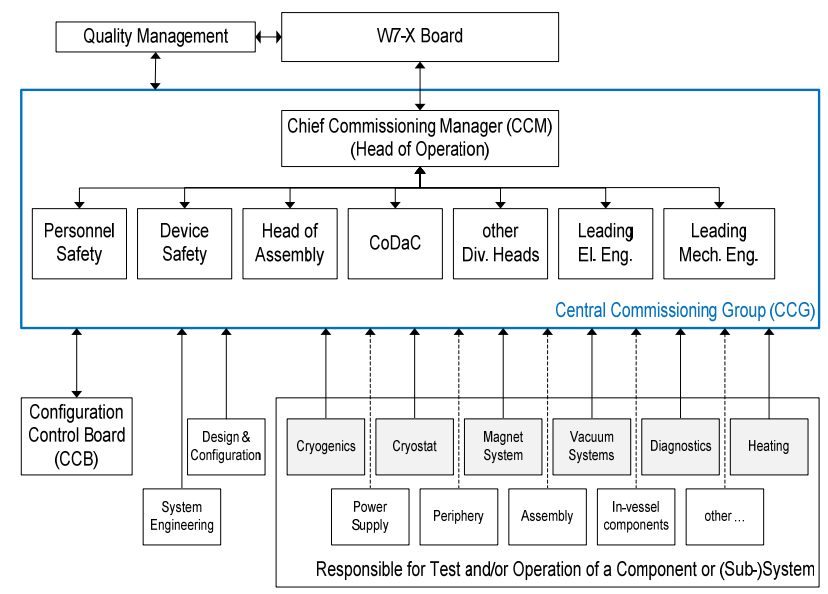

Fig. 1 Organizational structure for commissioning

The CCM leads the Central Commissioning Group (CCG). Permanent members of the CCG are the Personnel Safety Manager for the torus hall, the responsible for the device safety, and the heads of $\mathrm{CoDaC}$ and Assem- bly. The subdivision heads, responsible for the system currently under commissioning, are also members of the CCG to provide the full technical competence.

Urgent problems can be cleared also in meetings of the Configuration Control Board (CCB) in which the scientific requirements are represented by the Scientific Director (ScD) of W7-X. Strategic decisions are made in the W7-X Board.

The CCG is supported by staff members of the cryogenics, cryostat, magnet system, vacuum systems, diagnostics, and heating departments. They will ensure best flow of information between the CCG and the respective expert groups.

\section{Documents}

The required documents for preparation and execution of the commissioning shall be explained in more detail.

\subsection{WBS - work breakdown structures}

In preparation of commissioning an individual WBS is prepared for each system by the RO. The WBS must contain the below listed key points, either as milestones or as work packages:

- preparation of documentation,

- verification of provided hardware particularly from other systems or projects,

- verification of provided software,

- necessary inspections prior to start of tests or commissioning,

- simplified commissioning sequences (commissioning steps) based on the CAT

- final steps for verification and documentation.

This WBS is used to communicate the status of commissioning during the weekly meetings of the CCG.

\subsection{Instruction manual (operating manual)}

All systems must be accompanied by instructions which allow the (specialized) personnel to put it into service, to operate, and to service the system (machinery) (cf. Produktsicherheitsgesetz - ProdSG 2011 - Product Safety Act).

To accelerate the preparation of concurring instruction manuals, a draft with integrated guideline was created in the project centrally.

Most of the required information is usually already part of existing documents like technical, functional or project specifications, safety analyses, description of interfaces, work instructions, operation manuals of bought components, etc. The relevant information can be extracted from these documents, or the approved controlled documents can be cited or appended.

Instructions for the training of operators and for short-term maintenance must be added if necessary. 
The CCM approves the document. The involved staff members must be instructed before any commissioning or operation of the system. The instruction of the staff requires a written acknowledgment.

\subsection{Safety analysis}

Special care must be taken for personnel safety. Any hazards must be avoided. And also risks for the device during operation must be minimized. Already during the functional or project specification a safety analysis was created. But this typically describes only the hazards and risks during (later) operation of the system. Therefore a special safety (or hazard) analysis is necessary for two reasons:

On the one hand in this starting situation of commissioning sometimes the functionality from other systems is not yet provided fully. To compensate this sometimes additional safety precautions must be implemented like special mechanical protection, control of special work within designated areas, provision of spare parts, mobile safety devices (radio emergency onoff switches), additional monitoring and control functions, and if required, additional staff members.

On the other hand the system currently under commissioning itself has not been tested yet for all operation conditions and purposes. Therefore for this first commissioning phase often special precautions are necessary to secure safe operations for the staff and the machine. This is the issue of the special safety analysis for (first) commissioning.

Based on the instruction manual, the CAT and the safety analysis the Personnel Safety Manager prepares a special health and safety briefing for the staff. The involved staff members must be instructed before execution of commissioning steps adequately on a daily base for changing conditions reasons. The instruction of the staff members requires a written acknowledgment.

\subsection{CAT - commissioning assurance template - commissioning instructions}

For each commissioning the RO has to prepare a commissioning assurance template (CAT) as commissioning instruction, which contains all necessary work and test steps in the right sequence. The CAT is based on the technical specification of the system and the instruction manual as well as on the safety analysis.

The CAT is structured similar to the established QAAP during assembly and must imply the following essential points:

- preparation of function tests including determination of parameters which shall be achieved in accordance with the specification and other decisions (over load testing inclusively if applicable),

- definition of safety-tests,

- initial conditions and necessary prerequisites for the execution (hard- and software, other components or systems) - preparatory works,

- detailed work and test steps and sequences,
- responsibilities for each step,

- definition of hold points,

- applicable documents,

- corresponding checks to confirm the achieved states with instruction for documentation like test protocols and check lists.

The CAT fixes all the specified individual preparatory steps and test sequences in their required order. Details shall be refined to such an extent that the CAT provides sufficient information on relevant activities. Documents such as specifications, technical drawings, technological directives (TD), instruction manual, work instructions (WI) and test instructions (TI), which are required for the execution of individual activities, must be specified, and it shall be explained how they are to be drawn up e.g. as check lists, test protocol etc. Prior to the approval of the CAT these documents must be also in an approved state and published in the central documentation system.

Hold points regulate organizational and chronological interaction of the persons involved. Hold points shall always be set when work or test sequences have to be extra approved prior to start (declaration of readiness for other systems, approval after evaluation of results from previously made steps etc.).

On arriving at hold points also the current states shall be compared with previously planned states. The achieved operational states and relevant operational data must be recorded and submitted to the responsible person for signature.

The successful completion of each step as well as quality variances or any other deviations must be recorded by the responsible staff member.

Correct preparation and maintenance of CAT are of particular importance for the verification of system conformity and provides the basis for the following commissioning of other systems. All noteworthy observations, experiences, proposals for improvement and modifications shall be noted in the CAT to provide an improvement of all technical and organizational processes. The CCM approves the prepared CAT.

After execution of all CAT steps the completion will be checked at least by the CCM and the other permanent members of the CCG, and QM. Additional checks by other can be necessary. The CCM confirms with the approval of the document the serviceability of the relevant system. Prior to any further activities, a release confirmation must be issued to allow operating a system together with other systems for commissioning.

In addition the whole set of regulations regarding the quality management of $\mathrm{W} 7-\mathrm{X}$ is still valid in the project as before.

\subsection{Changes or repeated commissioning}

Changes will always have to be applied for, approved and carried out in compliance with the given rules for the 
execution of changes in the project for the following major activities:

1. Separation of systems which are scheduled for testing or commissioning (interfaces),

2. Changes to the specification of function tests including parameters which are to be achieved according to specification or other relevant requirements (overload tests inclusive),

3. Changes to the definition of safety inspections.

Operationally necessary and permissible changes of test sequences in accordance with CAT are allowed to be approved by the CCM in coordination with RO and must be recorded on CAT.

Commissioning must be repeated completely or partly if (single) function or safety tests could not be carried out successfully, or if the statement about the success or the serviceability of the relevant system is no longer valid because of additional changes or expansions. The existing CAT is the basis for the repetition of commissioning; however, the CAT must be adapted in general as required by the changed conditions. The CAT for the repetition of commissioning has to be checked and approved again according to the responsibilities.

\section{First experience during commissioning of the cryostat vessel}

The first commissioned system was the cryostat system. Fig. 2 shows, that several local and integrated commissioning of subsystems were necessary to test the serviceability of the cryostat vessel and the vacuum systems as one prerequisite for cooling down the magnet system inside the cryostat.

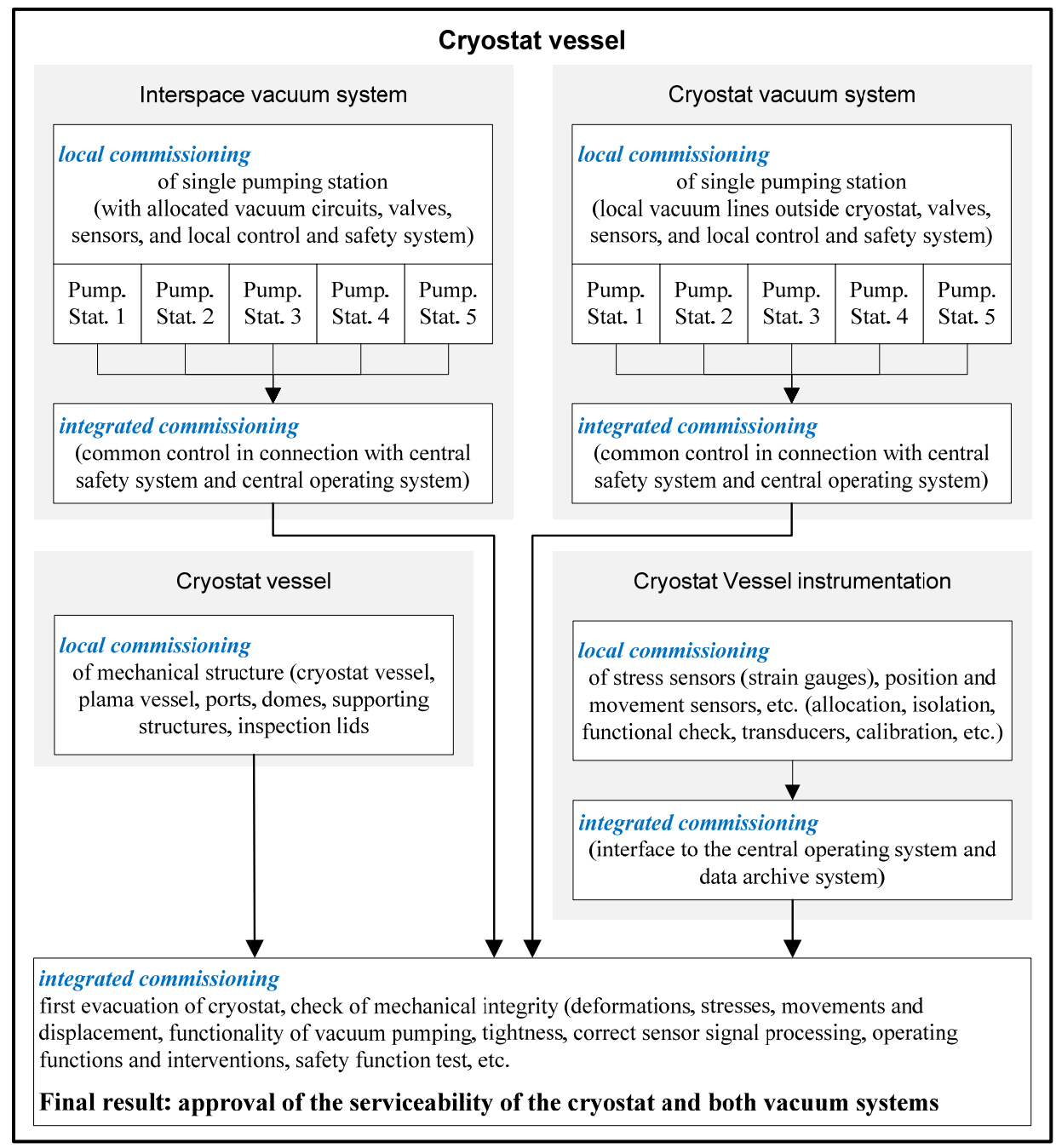

Fig. 2 commissioning sequence of the cryostat system (w/o cooling of magnets)

\subsection{Interspace vacuum system}

This system consists of five independent pumping stations for generating the vacuum for the multilayer bellows of W7-X and the double sealing gaskets. Multilayer bellows are used in about 40 ports between the plasma vessel and the outside of the cryostat to enable thermal and stress induced movements. In case there is vacuum on the outside of the bellows the mechanical stability is guaranteed only when the space between the layers are under vacuum.

For local commissioning five CAT were prepared, one for each pumping station. In this way the commissioning could be started for each system individually, even if last assembly work was to be done at the others. After initial 
check of all necessary prerequisites (hard- and software installation, electrical safety checks, mechanical completeness, etc.) the commissioning started with the stand alone test of the pumps in conjunction with their control. Only after these steps were completed successfully the vacuum circuits were switched on one by one to test the functionality stepwise. For each circuit the specified set of tests was carried out to confirm the proper function. For necessary rework the CAT sequence was interrupted for repair or adjustment work.

\subsection{Cryostat vacuum system}

The local and integrated commissioning of the cryostat vacuum system (five single pumping stations with valves and gate valves) was carried out in the same way principally.

\subsection{Cryostat vessel instrumentation}

As next required system the permanent cryostat vessel instrumentation was commissioned; first locally and subsequent integrally in connection with the central operating system and the data archive. Also some temporary instrumentation like metrology and an acoustic monitoring system were implemented to extend the capability of structural integrity assessment during first evacuation of the cryostat vessel.

\subsection{Cryostat vessel}

The local commissioning of the cryostat vessel covered mainly checks of the finalized assembly, the removal of temporary supports, etc.

The integrated commissioning of the cryostat vessel was the first real complex task. A special risk assessment was prepared to consider all the risks related to the first evacuation of a newly built vessel. This analysis resulted in an accurate definition of evacuation phases, permanently repeating tests, and personnel protection measures like restrictions of access. No risks were allowed. As shown in fig. 3 the evacuation was carried out in several pressure stages.

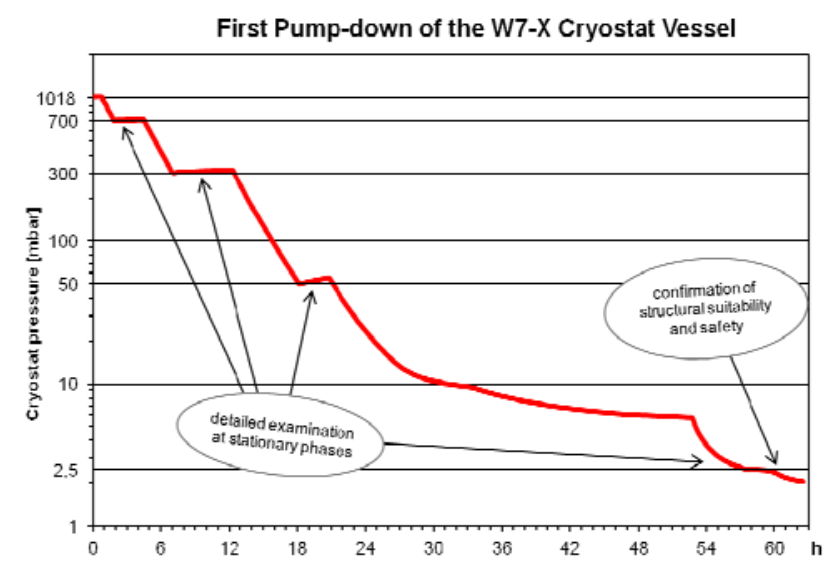

Fig. 3 First evacuation of the cryostat vessel with transient phases and detailed examinations during stationary phases [6]

The most significant sensors for assessment of the mechanical behavior were monitored and compared with the predicted values continuously during the transient phases. The process could have been immediately stopped in the case of irregular or not interpretable indications. When reaching a defined pressure stage the evacuation was interrupted and a systematic examination of the mechanical integrity was carried out on the basis of the collected sensor data and a very carefully visual inspection of the presumably weak points of the vessel like small weld seams or the regions of the bellows. After successful passing of these tests the evacuation was continued till the next pressure stage. In the end the inside pressure reached 2.5 mbar and after a last final examination the structural suitability and safety could be confirmed. To exclude any risks in the future for the next venting and evacuation actions some additional monitoring measures were fixed. The next step is the integral leak test of the weld seams and sealing gaskets which not have been tested yet.

\section{Summary}

Because quality management during commissioning of W7-X is a key issue, rules were defined for the organizational and technical approach, the preparation of the suitable documents and the realization of all commissioning steps. The first experiences during commissioning of the cryostat vessel show the fundamental suitability of the procedures, although in future still some elements will be improved or adapted.

\section{Acknowledgments}

All work as described above was done in close cooperation between the departments of W7-X, physicist, assembly and operation staff, project control and the QM-department. The good collaboration between these various partner, sometimes under strong time pressure, is gratefully acknowledged.

This project has received funding from the European Union's Horizon 2020 research and innovation program under grant agreement number 633053. The views and opinions expressed herein do not necessarily reflect those of the European Commission.

\section{References}

[1] J.L. Hemmerich, et. al., Installation and inactive commissioning of the JET active gas handling system (AGHS), in: 15. IEEE/NPSS Symposium on Fusion Engineering Supplement, 1993, pp. 37-41.

[2] R. Saban, et. al., The commissioning of the LHC technical systems, in: IEEE Particle Accelerator Conference PAC, June 25-29, 2007, pp. 3801-3803,

[3] I. Kuehn, J.-J. Cordier, S. Chiocchio, B. Levesy, B. Manfreo, J. Reich, et. al., Progress in design integration and configuration control of the ITER machine baseline, in: 23rd IEEE/NPSS Symposium on Fusion Engineering, SOFE 2009, pp. 1-4

[4] Minutes of Workshop Commissioning of W7-X, Greifswald, January 16 - 18, 2013, W7-X-Doc-SystemNo. 1-JBA02-C0001

[5] R. Vilbrandt, Wendelstein 7-X Team; Quality assurance during assembly of Wendelstein 7-X, Fusion Eng. Des. 86, (2011) 655-658

[6] H.-St. Bosch, et. al.; Experience with the Comissioning of the Superconducting Stellarator W7-X, 2015 (this conference) 